\title{
STEVIA MANDONII (ASTERACEAE,EUPATORIEAE): PRIMER REGISTRO PARA LA FLORA ARGENTINA Y LECTOTIPIFICACIÓN
}

\author{
Juan F. Rodríguez-Cravero', Mariana A. Grossi' ${ }^{2,3}$ \& Diego G. Gutiérrez ${ }^{1,3}$
}

\begin{abstract}
${ }^{1}$ División Plantas Vasculares, Museo Argentino de Ciencias Naturales (MACN-CONICET), Av. Ángel Gallardo 470, C1405DJR Ciudad de Buenos Aires, Argentina; jfrc@macn.gov.ar (autor corresponsal).

${ }^{2}$ División Plantas Vasculares, Museo de La Plata (Facultad de Ciencias Naturales y Museo, Universidad Nacional de La Plata), Paseo del Bosque s.n., B1900FWA La Plata, Buenos Aires, Argentina. Consejo Nacional de Investigaciones Cientificas y Técnicas (CONICET).

${ }^{3}$ Laboratorio de Morfología Comparada de Espermatófitas (LAMCE, Facultad de Ciencias Agrarias y Forestales, Universidad Nacional de La Plata), Av. 60 s.n. entre 116 y 118, B1900FWA La Plata, Buenos Aires, Argentina.
\end{abstract}

\begin{abstract}
Rodríguez-Cravero, J. F.; M. A. Grossi \& D. G. Gutiérrez. 2020. Stevia mandonii (Asteraceae, Eupatorieae): First record for the Argentine flora and lectotypification. Darwiniana, nueva serie 8(1): 402-409.

Stevia Cav. (Asteraceae, Eupatorieae) includes ca. 235 species distributed from United States of America to Chile and Argentina in South America. During the taxonomic revision of the Argentinian species several specimens belonging to Stevia mandonii Sch. Bip. were found in the province of Jujuy, where it had not been previously cited. In this framework, the goals of the present article are 1) to report the species in Argentina for the first time, 2) to differentiate it among morphologically close species, and 3) to perform a taxonomical analysis based on herbarium specimens, populations in the field, bibliography and type material. Morphology was analyzed by means of optical microscopy and scanning electron microscopy techniques. Stevia mandonii is readily distinguished by its small size of 10-20 cm, rounded leaves and pappus formed by 9-17 bristles with presence of heterocarpy. A detailed morphological description, an illustration, a geographic distribution map, and a key to species of Stevia that occur in Jujuy are provided. The designation of a lectotype for the name Stevia mandonii is properly discussed.
\end{abstract}

Keywords. Andes; Bolivia; Compositae; Jujuy; Perú; Stevia; taxonomy.

Resumen. Rodríguez-Cravero, J. F.; M. A. Grossi \& D. G. Gutiérrez. 2020. Stevia mandonii (Asteraceae, Eupatorieae): Primer registro para la flora argentina y lectotipificación. Darwiniana, nueva serie 8(1): 402-409.

Stevia Cav. (Asteraceae, Eupatorieae) incluye ca. 235 especies distribuidas de Estados Unidos de América a Chile y Argentina en Sudamérica. Durante la revisión de las especies argentinas se halló material correspondiente a Stevia mandonii Sch. Bip. en el noroeste del país, donde no había sido citada previamente. En este marco, los objetivos del trabajo son 1) registrar por primera vez a $S$. mandonii para la Argentina, 2) distinguirla de las especies afines morfológicamente y 3) llevar a cabo un estudio taxonómico basado en ejemplares de herbario, poblaciones en el campo, bibliografía e imágenes de alta resolución del material tipo. La morfología se analizó por medio de técnicas de microscopía óptica y microscopía electrónica de barrido. Stevia mandonii se distingue morfológicamente por su tamaño pequeño, de 10-20 cm de alto, hojas ovadas o redondeadas con ápice obtuso y papus formado por 9 a 17 aristas con presencia de heterocarpía. Como resultado se provee una descripción morfológica detallada, una ilustración, un mapa de distribución y una clave para las especies de Stevia presentes en la provincia de Jujuy. Además, se discute la designación de un lectotipo para el nombre Stevia mandonii.

Palabras clave. Andes; Bolivia; Compositae; Jujuy; Perú; Stevia; taxonomía. 


\section{INTRODUCCIÓN}

Stevia Cav. es un género americano constituido por ca. 235 especies (King \& Robinson, 1987; Soejima et al., 2001; Quaresma et al., 2013; Turner, 2013a; 2013b; 2015; Rodríguez-Cravero et al., 2017a; 2019) que se caracterizan por presentar hábito herbáceo o arbustivo, involucro cilíndrico, eximbricado, con cinco filarios, cinco flores por capítulo, un anillo de tricomas erectos en el interior de la corola a nivel de la garganta, cipselas 5-costadas y un papus formado mayoritariamente por escamas y aristas (Robinson, 1930; Cabrera, 1978; King \& Robinson, 1987). Se estima que al menos un tercio de las especies de Stevia presentan heterocarpía (Robinson, 1930), es decir, cuatro cipselas (adelfocarpos) con un tipo de papus y la quinta (idiocarpo) con un papus ausente o reducido por capítulo (King \& Robinson, 1987; Hind \& Robinson, 2007).

En el continente americano las especies de Stevia poseen un amplio rango de distribución, extendiéndose desde el sur de los Estados Unidos de América y norte de México hasta el norte de Chile y la Patagonia argentina (King \& Robinson, 1987; Freire \& Ariza Espinar, 2014; Gutiérrez et al., 2016). En Argentina se registran 33 especies distribuidas principalmente en el noroeste del país (NOA: Catamarca, Jujuy, La Rioja, Salta y Tucumán), una de las áreas con mayor riqueza de especies de plantas vasculares y de Asteraceae en particular (Katinas et al., 2007; Grossi et al., 2012). En esta región, Stevia presenta 25 especies, 22 de ellas endémicas (Cabrera, 1978; Freie \& Ariza Espinar, 2014) que ocupan ambientes áridos de la Puna y Prepuna, en las altas cumbres de la Cordillera de los Andes y en las selvas húmedas de las Yungas (Freire et al., 2006; Freire, 2008; Freire \& Ariza Espinar, 2014; Rodríguez-Cravero et al., 2017b). En un viaje de colección reciente al NOA (2019) se hallaron poblaciones de Stevia mandonii Sch. Bip., una especie que no había sido citada hasta el momento para el país, y que se registra para la provincia de Jujuy (Mina El Aguilar y Abra de Lizoite). Stevia mandonii puede ser confundida morfológicamente en base a su hábito y morfología foliar y floral con las especies andinas S. chamaedrys Griseb., S. maimarensis (Hieron.) Cabrera, S. mercedensis Hieron., S. minor Griseb. y $S$. tarijensis Hieron.
En este marco, el objetivo del presente artículo es registrar por primera vez a Stevia mandonii para la Argentina, describir su morfología, proporcionar una clave dicotómica para diferenciarla de las especies morfológicamente afines y lectotipificar el nombre del taxón.

\section{MATERIALES Y MÉTODOS}

El material estudiado fue coleccionado en los departamentos de Humahuaca y Yavi en la provincia argentina de Jujuy y depositado en el herbario BA (acrónimo de acuerdo a Thiers, 2019). La identificación taxonómica se llevó a cabo mediante un análisis morfológico y la comparación de los tipos nomenclaturales con el protólogo correspondiente. Para el análisis del material tipo de $S$. mandonii se utilizaron las imágenes digitales disponibles en JSTOR Plant Science (https:// plants.jstor.org). La información del protólogo de $S$. mandonii fue contrastada con los ejemplares originales y los datos observados en las etiquetas. Además, se estudiaron especímenes tipo y no tipo alojados en los herbarios BA, BR, F, GH, GOET, K, LP, MPU, NY y S (Thiers, 2019), tanto para acciones nomenclaturales como para interpretar la circunscripción de la especie y sus afines.

Los caracteres diagnósticos utilizados para la identificación del taxón fueron: hábito, longitud de los entrenudos, tamaño y forma de la lámina foliar, tipo de inflorescencia secundaria (capitulescencia), tamaño y forma de los filarios, pubescencia, tamaño, forma y color de las flores, pubescencia y tamaño de las cipselas, elementos y tipo de papus y la presencia de heterocarpía. Para la descripción de los tricomas se siguió la terminología de Ramayya (1962), excepto para los pelos gemelos donde se siguió a Hess (1938), Freire \& Katinas (1995) y Sancho \& Katinas (2002). La descripción del margen de la lámina foliar y las cipselas se basó en la terminología propuesta por Metcalfe \& Chalk (1950) y Harris \& Harris (1994). Las observaciones y fotografías se realizaron con un microscopio óptico Nikon Eclipse E200 y una lupa binocular Nikon SMZ1000. Las fotografías al microscopio electrónico de barrido fueron tomadas con un Philips XL-30 SEM (Museo Argentino de Ciencias Naturales C.A.B.A., Argentina). 


\section{RESULTADOS}

Stevia mandonii es afín a otras especies del género presentes en el NOA que poseen capítulos sésiles (o brevemente pedunculados) y láminas foliares menores a $6 \mathrm{~cm}$ de longitud. En este grupo de especies la forma de las láminas puede ser linear, elíptica u ovada, siendo frecuente que las hojas jóvenes presenten morfologías intermedias y generen dificultades para la correcta identificación de las especies. Por lo tanto, resulta necesario emplear otros caracteres morfológicos, como ser el tipo de papus, la altura de la planta y el ápice de la lámina foliar para circunscribir los taxa presentes en Jujuy. A continuación, se presenta una clave para la diferenciación de $S$. mandonii y las especies afines $S$. chamaedrys, $S$. maimarensis, $S$. mercedensis, S. minor y $S$. tarijensis.

\section{Clave para diferenciar Stevia mandonii de las especies afines presentes en Jujuy, Argentina}

1. Adelfocarpos con papus formado por 1-7 aristas, o papus isomórfico formado por 1-7 escamas ........................... 2

1. Adelfocarpos con papus formado por 8-21 aristas

2(1). Hojas no agrupadas en los nudos, láminas foliares elípticas u ovado-rómbicas, con margen crenado, aristas de menor longitud que la longitud de la corola S. chamaedrys

2. Hojas densamente agrupadas en los nudos, láminas foliares lineares u oblongas, con margen entero o apenas aserrado, aristas de igual longitud que la longitud de la corola S. minor

3(1). Láminas foliares lineares u oblongas, con ápice agudo 4

3. Láminas foliares elípticas, ovadas o redondeadas, con ápice obtuso o subagudo .................................................. 5 4(3). Láminas foliares oblongas, de hasta $6 \mathrm{~cm}$ de longitud; papus formado por 8-12 aristas 4. Láminas foliares lineares, de hasta $3,3 \mathrm{~cm}$ de longitud; papus formado por 13-21 aristas S. mercedensis 5(3). Plantas de 20-30 cm de alto; láminas foliares elípticas, ápice subagudo, glabras S. maimarensis 5. Plantas de10-20 cm de alto; láminas foliares ovadas u redondeadas, a veces angostamente ovadas en las ramas terminales, ápice obtuso, con pelos glandulosos

S. mandonii

\section{TRATAMIENTO TAXONÓMICO}

Stevia mandonii Sch. Bip., Linnaea 34: 535. 1866. Stevia mandonii Sch. Bip., Bull. Soc. Bot. France 12: 81. 1865, nom. nud. Stevia mandonii Sch. Bip. ex B.L. Rob., Contr. Gray Herb. 77: 6. 1926, nom. illeg. TIPO: Bolivia, Depto. La Paz, Prov. Larecaja, "viciniis Sorata, in collibus, prope Ancohuma, 3800 m", III/IV-1860, G. Mandon 246 (lectotipo NY 00260186!, aquí designado; isolectotipos, BR 00000553359!, F 14764!, F 911397!, GH 00012855!, GH 00012856!, GOET 002062!, K 000488815!, MPU 013128!, MPU 013130!, NY 00260187!, NY 00260188!, NY 00260189 !, S no. R-5941!). Figs. 1 y 2.

Hierbas perennes de $10-20 \mathrm{~cm}$ de altura, con xilopodio y múltiples tallos. Tallos hexagonales, costados, glanduloso-pubescentes, apenas ramificados cerca de la base y frondosos hasta la inflorescencia, entrenudos de 0,2-1 cm de long.
Hojas opuestas, sésiles; lámina ovada o redondeada, a veces angostamente ovada en las ramas terminales, de 0,9-2 × 0,4-1 cm, ápice obtuso, base aguda, margen generalmente entero o serrulado con un par de dientes cerca del ápice, trinervadas, punteado-glandulosas en el haz y el envés. Inflorescencias densas, dispuestas en 1-2 pares de ramas terminales, formando una cima corimbiforme; capítulos 5-20, densamente agrupados, sésiles, o raramente pedunculados, pedúnculos de $0,2 \mathrm{~mm}$ de long., densamente pubescentes con pelos no glandulares (simples cónicos y cilíndricos) y pelos glandulares sésiles (biseriados vesiculares subtipo $\beta$ ), ocasionalmente pelos glandulares pedunculados (biseriados vesiculares subtipo $\alpha$ ). Involucros de 4,5-6 × 2-4 $\mathrm{mm}$; filarios lineares, ovados $\mathrm{u}$ obovados, de 4-6 × 1-1,8 mm, los externos 2, de ápice agudo, pubescentes con pelos no glandulares y pelos glandulares sésiles, los internos 3, de ápice agudo $\mathrm{u}$ obtuso, glabros o ligeramente pubescentes. 


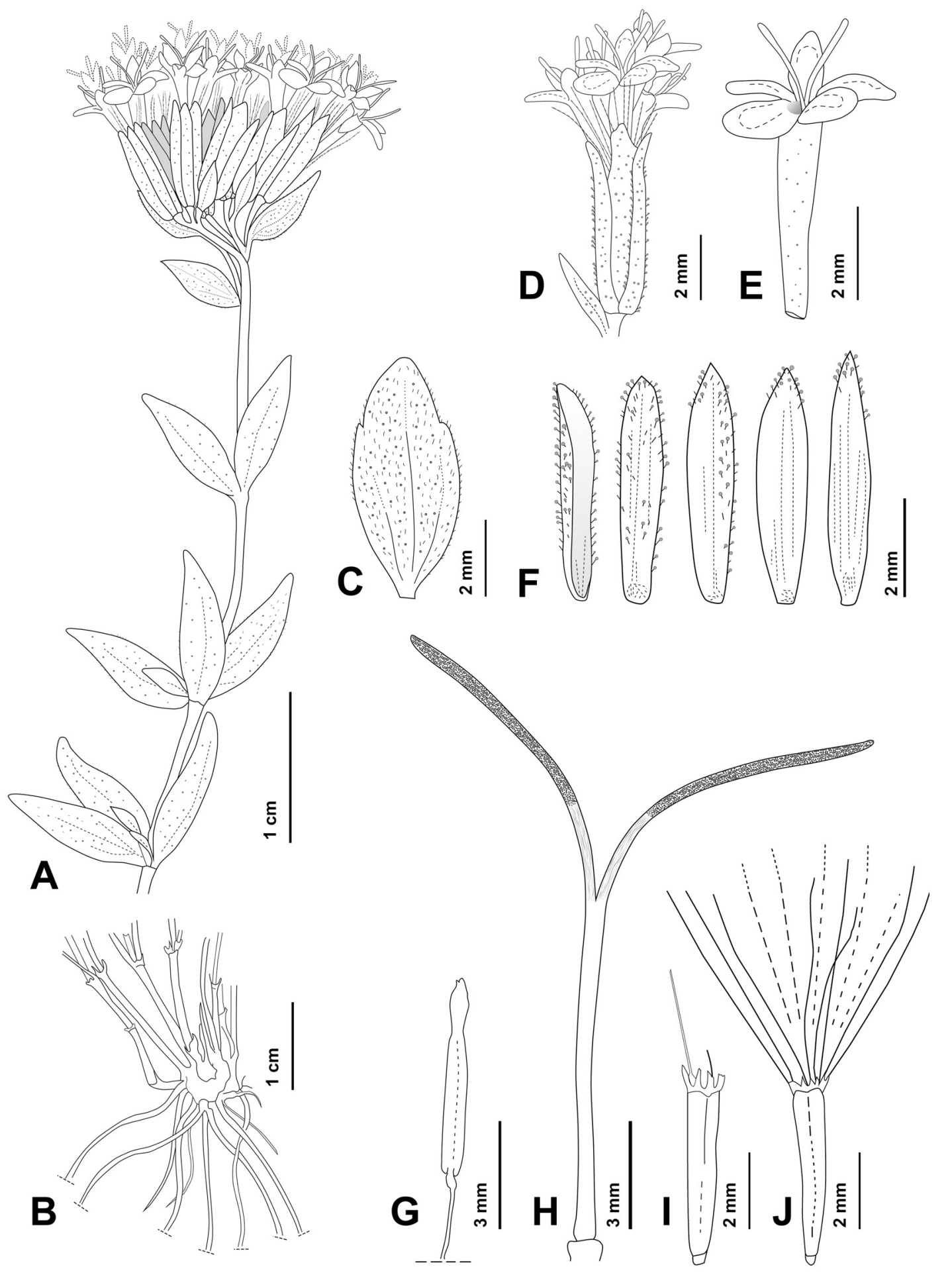

Fig. 1. Stevia mandonii Sch. Bip. A, rama florífera. B, xilopodio. C, hoja. D, capítulo. E, flor. F, filarios. G, antera. H, estilo. I, idiocarpo. J, adelfocarpo. Gutiérrez 1538 (BA). 


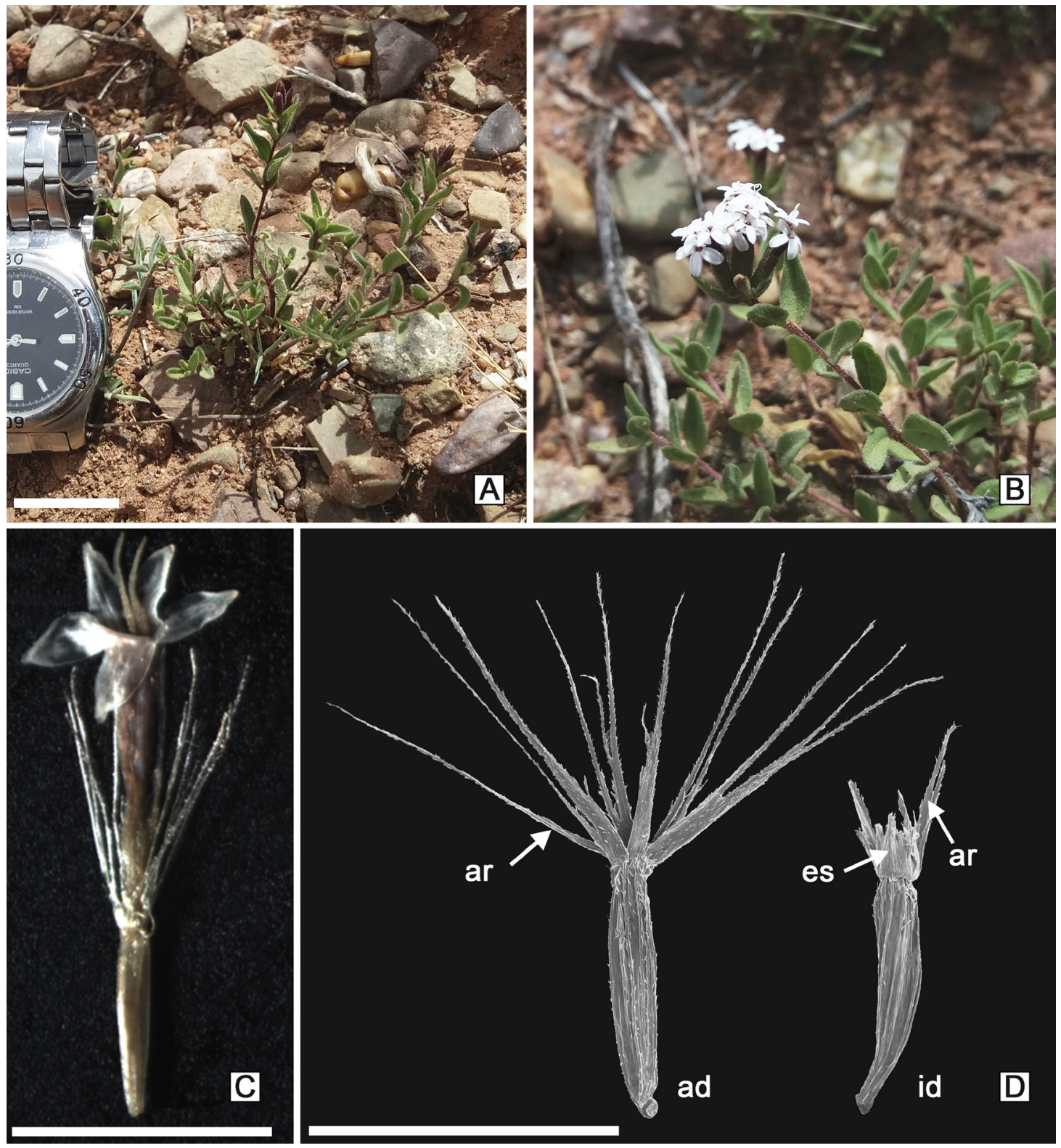

Fig. 2. Stevia mandonii Sch. Bip. A, aspecto general de la planta. B, detalle de hojas, capítulos y flores. C, flor (vista en lupa). D, cipselas con papus. Abreviaturas: ad, adelfocarpo; ar, arista; es, escama; id, idiocarpo (vistas con microscopio electrónico de barrido). Escalas: A: $2 \mathrm{~cm}$. C, D: $5 \mathrm{~mm}$. Fotos: Diego G. Gutiérrez (A-B). Figura en color en la versión en línea http://www.ojs.darwin.edu.ar/index.php/darwiniana/article/view/874/1184

Flores con corolas infundibuliformes, de 6-7,5 × 0,8$1,2 \mathrm{~mm}$, tubo rosado o violeta, de 4,6-6,5 $\mathrm{mm}$ de long., superficie externa pubescente, con pelos glandulares sésiles abundantes en el tercio inferior y pelos no glandulares, lóbulos 1-2 ×0,8-1,2 mm, ápice subagudo, blancos o rosados; estambres con anteras de 1,8-4,5 $\times$ 0,3-0,4 mm, apéndices conectivales obovados, ca. 0,4 $\times 0,2 \mathrm{~mm}$, margen laciniado, base de las tecas obtusas, collares anterales oblongos, de 0,15-0,21 mm de long.; estilos 7-11 mm long., ramas estilares ca. $4 \mathrm{~mm}$ long. 
Cipselas prismáticas o elipsoides, 2,3-3,9 × 0,8$1,2 \mathrm{~mm}$, pubescentes con pelos gemelos y pelos glandulares sésiles. Papus dimórfico, adelfocarpos con papus formado por 9-17 aristas, usualmente fusionadas en la base, 4,8-6 $\mathrm{mm}$ de long., e idiocarpos con papus formado por 5-7 escamas, 0,4-1,2 mm de long., y 1-2 aristas cortas, de 1,7$4 \mathrm{~mm}$ de long., estando todos los elementos del papus fusionados por sus bases (corona).

Distribución y hábitat. Stevia mandonii (Figs. 1 y 2) se distribuye en Perú (Cajamarca, Cuzco y Puno), Bolivia (Chuquisaca, Cochabamba, La Paz, Potosí y Tarija) y el noroeste de Argentina (Jujuy) (Fig. 3). Los nuevos registros de S. mandonii para la Argentina provienen de los departamentos de Cochinoca, Humahuaca, Rinconada y Yavi, donde habita entre rocas o en suelos arenosos-pedregosos, solitaria o asociada a vegetación herbácea $\mathrm{y}$ arbustos dispersos, en quebradas, cauces de ríos o laderas, a $3450-4500 \mathrm{~m}$ s.m.

Nota nomenclatural. Stevia mandonii fue designada por primera vez por C. H. Schultz "Bipontinus" (1865) en un listado de especies basado en ejemplares coleccionados por G. Mandon en Bolivia, refiriéndose al material original como: "246 et 246 bis. - Mandonii Sz Bip. aff. St. frigidae". Al momento de la designación por parte de Schultz el nombre careció de una diagnosis y por lo tanto constituye un nombre inválido (Turland et al., 2018). Posteriormente, el autor realizó una descripción formal de la especie en cuestión y de esta manera publicó válidamente el nombre (Schultz "Bipontinus", 1866). En este último trabajo declaró "246 et 246b. - Mandonii Sz. Bip., n. sp., affinis St. frigidae Karsten! sed omnium humillima; pappo 16-20-setoso, unius achenii vero abortivo", refiriéndose a plantas pequeñas con adelfocarpos formados por 16 a 20 aristas del papus y un idiocarpo. Posteriormente, Robinson (1926) consideró que los nombres $S$. mandonii en los trabajos de Schultz "Bipontinus" carecían de una descripción morfológica (i.e., "without char" [sic]). Hacia finales del siglo XX el criterio taxonómico de Robinson no fue seguido por King \& Robinson (1987). Recientemente, Hind (2011) retomó el enfoque taxonómico de Robinson (1926).

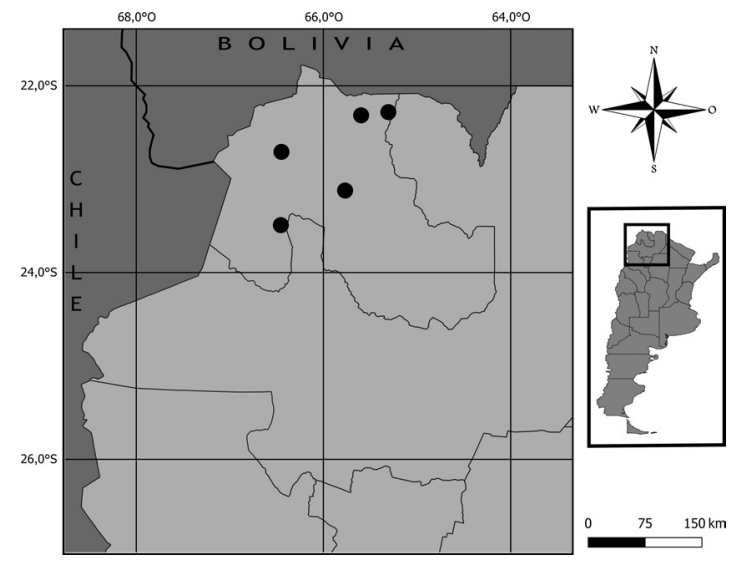

Fig. 3. Distribución de Stevia mandonii en Jujuy, Argentina.

Finalmente, Robinson (2014) citó la especie en Bolivia como $S$. mandonii Sch. Bip. asignándole por primera vez el sinónimo $S$. mandonii Sch. Bip. ex B.L. Rob.

Respecto al material original asociado a los nombres publicados por Schultz "Bipontinus", las colecciones del autor se encuentran depositadas principalmente en el herbario $\mathrm{P}$ y secundariamente en BM, BR, CGE, FI, FR, H. K, L, LI, MANCH, MW, OXF, PH, REG y W (Stafleu \& Cowan, 1985). Los ejemplares tipo de Mandon depositados en B fueron destruidos durante la Segunda Guerra Mundial aunque, afortunadamente, existen duplicados del material original distribuidos por numerosos herbarios del mundo (Stafleu \& Cowan, 1985). Ante la ausencia de la designación de holotipo por parte de Shultz, resulta necesario designar un lectotipo (Turland et al., 2018; McNeill, 2014). Tras el análisis de los sintipos "Mandon 246" depositados en los herbarios BR (00000553359), F (14764 y 911397), GH (00012855 y 00012856), GOET (002062), K (000488815), MPU (013128 y 013130), NY (00260186, 00260187, 00260188 y 00260189) y $\mathrm{S}$ (R-5941), y no habiendo encontrado ejemplares depositados en $\mathrm{P}$, se designa de acuerdo al CIN (Turland et al., 2018) el ejemplar NY 00260186 como lectotipo del nombre $S$. mandonii debido a que el material se ajusta adecuadamente a la descripción original y posee numerosas hojas y capítulos correctamente preservados, que son de valor diagnóstico para la identificación del taxón. 


\section{Material examinado.}

ARGENTINA. Jujuy. Dpto. Cochinoca, Corral Negro a Ciénaga Grande, 4-II-1937 (fl, fr), Castellanos 20235 (LP). Dpto. Humahuaca, Cerro Aguilar, 23-II-1953 (fl, fr), Hunziker \& Cano 6165 (LP); Ciénaga de Zarzo, 11-III-1967 (fl, fr), Fabris \& Crisci 6875 (LP); Mina El Aguilar, río Despensa, $23^{\circ} 2^{\prime} 11,80^{\prime \prime} \mathrm{S}-65^{\circ} 38^{\prime} 24,20^{\prime \prime} \mathrm{O}, 1-\mathrm{III}-2019$ (fl, fr), Gutiérrez et al. 1531 (BA). Dpto. Rinconada, Mina Pirquitas, 1-III-1964 (fl, fr), Schwabe et al. 700 (LP), 1115 (LP). Dpto. Yavi, Quebrada de Cajas, 21-II-1971 (fl, fr), Cabrera et al. 21528 (LP); Abra de Lizoite, $22^{\circ} 15^{\prime} 16,5^{\prime \prime} \mathrm{S}-65^{\circ} 07^{\prime}$ $18,1^{\prime \prime} \mathrm{O}$, próximo al límite interprovincial JujuySalta, camino a Santa Victoria Oeste, 1-III-2019 (fl, fr), Gutiérrez et al. 1538, 1542 (BA).

\section{AGRADECIMIENTOS}

Los autores agradecemos a los tres revisores anónimos por todas las sugerencias realizadas sobre el manuscrito, al igual que a Gonzalo J. Marquez (UNLP) por su ayuda en el viaje de campo, a Mónica L. Stampacchio (MACN) por la preparación de los ejemplares de herbario y a Fabián Tricárico por su asistencia técnica en el MEB. Asimismo, agradecemos a la curadora del Herbario LP, Laura Iharlegui, por proporcionar el acceso a parte del material examinado. Este trabajo fue financiado por el Consejo Nacional de Investigaciones Científicas y Técnicas (CONICET; PUE 22920160100098CO), la Agencia Nacional de Promoción Científica y Tecnológica (PICT-2017-0965) y la Universidad Nacional de La Plata (UNLP N814).

\section{BIBLIOGRAFÍA}

Cabrera, A. L. 1978. Stevia (Compositae). En A. L. Cabrera (ed.), Flora de la Provincia de Jujuy. Colección Científica del Instituto Nacional de Tecnología Agropecuaria 13(10): 58-89. Buenos Aires: INTA.

Freire, S. E. 2008. Stevia. En Zuloaga, F. O.; O. Morrone \& M. J. Belgrano (eds.). Catálogo de las Plantas Vasculares del Cono Sur (Argentina, Sur de Brasil, Chile, Paraguay y Uruguay). Monographs in Systematic Botany from the Missouri Botanical Garden 107 (2): 1509-1517.

Freire, S. E. \& L. Katinas. 1995. Morphology and ontogeny of the cypsela hairs of Nassauviinae (Asteraceae, Mutisieae).
En D. J. N. Hind, C. Jeffrey \& G. V. Pope (eds.), Advances in Compositae Systematics 107-143. Kew: Royal Botanical Gardens.

Freire, S. E.; E. Urtubey, G. Sancho, N. D. Bayón, L. Katinas, D. G. Gutiérrez, D. A. Giuliano, A. A. Sáenz, L. Iharlegui \& G. Delucchi. 2006. Inventario de la biodiversidad vegetal de la provincia de Misiones: Asteraceae. Darwiniana 44: 375-452.

Freire, S. E \& L. Ariza Espinar. 2014. Stevia, en F. O. Zuloaga, M. J. Belgrano \& A. M. Anton (eds.), Flora Argentina: Flora Vascular de la República Argentina, vol. 7(1): 409435. Buenos Aires: Estudio Sigma SRL.

Grossi, M. A.; D. G. Gutiérrez \& G. Delucchi. 2012. Una mirada sobre el estado actual de la conservación de la flora argentina. Conservación Vegetal 16: 15-17.

Gutiérrez, D. G.; M. Muñoz-Schick, M. A. Grossi, J. F. Rodríguez-Cravero, V. Morales \& A. Moreira-Muñoz. 2016 The genus Stevia (Eupatorieae, Asteraceae) in Chile: a taxonomical and morphological analysis. Phytotaxa 282: 1-18. DOI: https://doi.org/10.11646/phytotaxa.282.1.1

Harris, J. G. \& M. W. Harris. 1994. Plant identification terminology: An illustrated glossary. Spring Lake: Spring Lake Publishing.

Hess, H. 1938. Vergleichende Untersuchungen über die Zwillingshaare der Kompositen. Botanische Jahrbücher für Systematik, Pflanzengeschichte und Pflanzengeographie. 68: 435-496.

Hind, D. J. N. 2011. An annotated preliminary checklist of the Compositae of Bolivia, version 2. Disponible en: http://www. kew.org/science/tropamerica/boliviacompositae/checklist.pdf [consulta noviembre de 2019].

Hind, D. J. N. \& H. Robinson. 2007. Eupatorieae, en K. Kubitzki (ed.). The families and genera of vascular plants, vol. 8: 510-574. Berlin: Springer.

Katinas, L.; D. G. Gutiérrez, M. A. Grossi \& J. V. Crisci. 2007. Panorama de la familia Asteraceae (Compositae) en la República Argentina. Boletín de la Sociedad Argentina de Botánica 42: 113-129.

King, R. M. \& H. Robinson. 1987. The genera of the Eupatorieae (Asteraceae). Monographs in Systematic Botany from the Missouri Botanical Garden 22: 1-581.

McNeill, J. 2014. Holotype specimens and type citations: General issues. Taxon 63: 1112-1113. DOI: https://doi.org/10.12705/635.7

Metcalfe, C. R. \& L. Chalk. 1950. Anatomy of the Dicotyledons. Vol. II. Oxford: Clarendon Press.

Quaresma, A. S.; J. N. Nakajima \& N. Roque. 2013. Stevia grazielae (Asteraceae: Eupatorieae: Ageratinae): a new species from the Cadeia do Espinhaço, Minas Gerais, Brazil. Kew Bulletin 68: 647-650. DOI: https://doi.org/10.1007/ s12225-013-9483-8 


\section{J. F. RODRÍGUEZ-CRAVERO ET AL. Stevia mandonii: Primer registro para la Flora Argentina}

Ramayya, N. 1962. Studies on the trichomes of some Compositae I. General structure. Bulletin of the Botanical Survey of India 4: 177-188.

Robinson, B. L. 1926. Records preliminary to a general treatment of the Eupatorieae, VI. Contributions from the Gray Herbarium of Harvard University 77: 3-62.

Robinson, B. L. 1930. Observations on the genus Stevia (The Stevias of the Argentine Republic; The Stevias of Paraguay; The Stevias of North America). Contributions from the Gray Herbarium of Harvard University 90: 36-160.

Robinson, H. 2014. Stevia. En P. M. Jørgensen, M. H. Nee \& S. G. Beck (eds.) Catálogo de las plantas vasculares de Bolivia. Monographs in Systematic Botany from the Missouri Botanical Garden 127: 369-372.

Rodríguez-Cravero, J. F.; D. G. Gutiérrez \& L. Katinas. 2017a. Lectotypifications in Uruguayan Stevia (Asteraceae, Eupatorieae). Phytotaxa 291: 116-122.

Rodríguez-Cravero, J. F.; M. A. Grossi, T. FuentesCastillo \& D. G. Gutiérrez. 2017b. Cambio climático y modelado de distribución de especies de Stevia (Asteraceae) en el noroeste de Argentina. Ecología Austral 27: 462-473.

Rodríguez-Cravero, J. F.; D. G. Gutiérrez, L. Katinas, M. A. Grossi, J. M. Bonifacino \& E. Marchesi. 2019. A revision and morphological analysis of the Uruguayan species of Stevia (Compositae, Eupatorieae). Rodriguésia 70: e01532018. 2019. DOI: http://dx.doi.org/10.1590/21757860201970078

Sancho, G. \& L. Katinas. 2002. Are the trichomes in corollas of Mutisieae (Asteraceae) really twin hairs? Botanical Journal of the Linnean Society 140: 427- 433.
Schultz "Bipontinus", C. H. 1865. Premièr listes de plantes des Andes boliviennes et distribuées par M. G. Mandon. Bulletin de la Société Botanique de France 12: 73-81.

Schultz "Bipontinus", C. H. 1866. Enumeratio Cassiniacearum a cl. G. Mandon in Bolivia a. 1857-1861 lectarum. Linnaea 34: 527-536.

Soejima, A.; T. Yahara \& K. Watanabe. 2001. Thirteen new species and two new combinations of Stevia (Asteraceae: Eupatorieae) from Mexico. Brittonia 53: 377-395. DOI: https://doi.org/10.1007/BF02809793

Stafleu, F. A. \& R. S. Cowan. 1985. Taxonomic Literature ( $2^{\circ}$ edición). Bohn, Scheltema \& Holkema, Utrecht. Disponible en: http://www.sil.si.edu/DigitalCollections/tl-2/ [consulta noviembre de 2019].

Thiers, B. [permanentemente actualizado, consulta noviembre 2019] Index Herbariorum: a global directory of public herbaria and associated staff. New York Botanical Garden's Virtual Herbarium, http://sweetgum.nybg.org/ih

Turland, N. J.; J. H. Wiersema, F. R. Barrie, W. Greuter, D. L. Hawksworth, P. S. Herendeen, S. Knapp, W. H. Kusber, D. Z. Li, K. Marhold, T. W. May, J. Mcneill, A. M. Monro, J. Prado, M. J. Price \& G. F. Smith. 2018. International Code of Nomenclature for algae, fungi, and plants (Shenzhen Code). Regnum Vegetabile 159.Glashütten: Koeltz Botanical Books. DOI: https://doi.org/10.12705/Code.2018

Turner, B. L. 2013a. Two new species of Stevia (Asteraceae: Eupatorieae) from Oaxaca, Mexico. Phytologia 95: 228-232.

Turner, B. L. 2013b. Stevia reinana (Asteraceae: Eupatorieae), a new species from near Yecora, Sonora, Mexico. Phytologia 95: 233-237.

Turner, B. L. 2015. Three new species of Stevia (Asteraceae: Eupatorieae) from northern Mexico. Phytologia 97: 25-27. 